\title{
Second-line therapy after nab-paclitaxel plus gemcitabine or after gemcitabine for patients with metastatic pancreatic cancer
}

\author{
E Gabriela Chiorean ${ }^{*}, 1$, Daniel D Von Hoff ${ }^{2}$, Josep Tabernero ${ }^{3}$, Robert El-Maraghi ${ }^{4}$, Wen Wee Ma ${ }^{5}$,
} Michele Reni ${ }^{6}$, Marion Harris ${ }^{7}$, Robert Whorf ${ }^{8}$, Helen Liu ${ }^{9}$, Jack Shiansong Li ${ }^{9}$, Victoria Manax ${ }^{9}$, Alfredo Romano 9 , Brian Lu' ${ }^{9}$ and David Goldstein ${ }^{10}$

\begin{abstract}
${ }^{1}$ Division Oncology, Department of Medicine, University of Washington, 825 Eastlake Avenue E, G4-833, Seattle, WA 98109-1023, USA; ${ }^{2}$ Translational Genomics Research Institute and HonorHealth, 445 North Fifth Street, Suite 600, Phoenix, AZ 85004, USA; ${ }^{3}$ Vall d'Hebron Institute of Oncology (VHIO), P Vall d'Hebron 119-129, Barcelona 08035, Spain; ${ }^{4}$ Royal Victoria Hospital Barrie Canada, 201 Georgian Drive, Barrie, Ontario, Canada L4M 6M2; ${ }^{5}$ Roswell Park Cancer Institute, 665 Elm Street, Buffalo, NY 14203, USA; ${ }^{6}$ San Raffaele Scientific Institute, Via Olgetina 60, 20132 Milan, Italy; ${ }^{7}$ Monash Health, 246 Clayton Road, Melbourne VIC 3168, Australia; ${ }^{8}$ Florida Cancer Specialists, 2401 60th Street Ct W, Bradenton, FL 34209-5500, USA; ${ }^{9}$ Celgene Corporation, 86 Morris Avenue, Summit, NJ 07901, USA and ${ }^{10}$ Department of Medical Oncology, Prince of Wales Hospital, South Sydney Illawarra, Barker Street, Sydney NSW 2031, Australia
\end{abstract}

Background: This exploratory analysis evaluated second-line (2L) therapy for metastatic pancreatic cancer in a large phase 3 trial (MPACT).

\begin{abstract}
Methods: Patients who received first-line (1L) nab-paclitaxel + gemcitabine (nab-P + Gem) or Gem were assessed for survival based on $2 \mathrm{~L}$ treatment received. Multivariate analyses tested influence of treatment effect and prognostic factors on survival.

Results: The majority of $2 \mathrm{~L}$ treatments (267 out of 347, 77\%) contained a fluoropyrimidine (5-fluorouracil or capecitabine). Median total survival ( $1 \mathrm{~L}$ randomisation to death) for patients who received $2 \mathrm{~L}$ treatment after $1 \mathrm{~L}$ nab-P + Gem vs Gem alone was 12.8 vs 9.9 months $(P=0.015)$. Median total survival for patients with a fluoropyrimidine-containing $2 \mathrm{~L}$ therapy after nab-P $+\mathrm{Gem}$ vs $\mathrm{Gem}$ was 13.5 vs 9.5 months $(P=0.012)$. Median $2 \mathrm{~L}$ survival (duration from start of $2 \mathrm{~L}$ therapy to death) was 5.3 vs 4.5 months for nab-P + Gem vs Gem, respectively $(P=0.886)$. Factors significantly associated with longer post- $1 \mathrm{~L}$ survival by multivariate analyses included $1 \mathrm{~L}$ nab-P + Gem, receiving $2 \mathrm{~L}$ treatment, longer $1 \mathrm{~L}$ progression-free survival, and Karnofsky performance status $\geqslant 70$ and neutrophil-to-lymphocyte ratio $\leqslant 5$ at the end of $1 \mathrm{~L}$ treatment.
\end{abstract}

Conclusions: These findings support the use of $2 \mathrm{~L}$ therapy for patients with metastatic pancreatic cancer. Fluoropyrimidinecontaining treatment after $1 \mathrm{~L}$ nab-P + Gem is an active regimen with significant clinical effect.

Pancreatic cancer is known for its aggressiveness, which is evident from mortality rates nearly equal to incidence (World Health Organization, 2015). Current estimates rank pancreatic cancer as the fourth leading cause of cancer-related mortality in the United States (American Cancer Society, 2015); however, it is projected to become the second leading cause of cancer death by 2030 (Rahib et al, 2014). Unfortunately, $\sim 80 \%$ of patients are initially diagnosed with advanced disease $(53 \%$ with metastatic disease and $28 \%$ with regional disease that has spread to lymph nodes (Surveillance, Epidemiology, and End Results Program, 2016)).

*Correspondence: Dr EG Chiorean; E-mail: gchiorea@uw.edu

Received 29 March 2016; revised 29 April 2016; accepted 16 May 2016; published online 28 June 2016

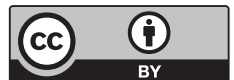


Treatment plans for advanced pancreatic cancer over the past decade have consisted primarily of only one line of therapy (Abrams et al, 2014; Smyth et al, 2015). This may have been, at least in part, due to the aggressive nature of the disease and the lack of effective treatment options.

Recent results from two phase- 3 trials (MPACT and PRODIGE) have demonstrated clinically meaningful, significantly longer overall survival (OS) with nab-paclitaxel + gemcitabine (nab-P + Gem) and FOLFIRINOX (folinic acid, 5-fluorouracil (5-FU), irinotecan, and oxaliplatin) regimens, respectively, vs Gem monotherapy, the standard for treating advanced pancreatic cancer since 1997 (Burris et al, 1997; Conroy et al, 2011; Von Hoff et al, 2013). The efficacy advantages observed for these new first-line (1L) therapies suggest greater opportunities for using second-line (2L) therapies in metastatic pancreatic cancer. The National Comprehensive Cancer Network currently recommends that if a Gem-based chemotherapy (such as with $n a b-\mathrm{P}+\mathrm{Gem}$ ) is used as $1 \mathrm{~L}$ therapy, the $2 \mathrm{~L}$ therapy should be a fluoropyrimidine-based regimen, including combination therapies for fit patients (The National Comprehensive Cancer Network, 2015).

A growing number of clinical trials are now examining the efficacy of $2 \mathrm{~L}$ therapy for metastatic pancreatic cancer, including the recent phase 3 NAPOLI- 1 study, which led to approval of MM398 in the 2L setting (Gill et al, 2014; Oettle et al, 2014; WangGillam et al, 2015). As nab-P + Gem has become a standard option in the $1 \mathrm{~L}$ setting, the identification of $2 \mathrm{~L}$ therapies that will be effective after 1L nab-P + Gem is important. This post-hoc analysis was designed to evaluate survival outcomes associated with $2 \mathrm{~L}$ treatment in patients enroled in the MPACT trial.

\section{PATIENTS AND METHODS}

Patients received $n a b-\mathrm{P}+\mathrm{Gem}$ or Gem alone as $1 \mathrm{~L}$ treatment for metastatic pancreatic cancer in the MPACT trial, as previously described (Von Hoff et al, 2013) Patients were treated until disease progression, unacceptable toxicity, or patient or physician decision. After treatment, OS and information on subsequent anticancer therapy were monitored on a monthly basis for 6 months and then every 3 months until death, study closure, or a period of 3 years had elapsed after treatment discontinuation, whichever occurred first. This evaluation was conducted by record review and/or telephone contact with the patient's treating physician. Data on subsequent therapies included only the type of treatment administered and the date that therapy was initiated. After the closure of the MPACT trial (NCT00844649) in 2013, an observational extension study was initiated to gather additional survival information on patients who were still alive (NCT02021500). The data collected from the extension study are included in this post-hoc evaluation.

Overall survival was analysed by the Kaplan-Meier method. Total OS was defined as time from 1L randomisation to death. For comparisons of patients who did $v s$ did not receive $2 \mathrm{~L}$ treatment, analyses were performed on post-1L OS, which was defined as the survival time from the end of $1 \mathrm{~L}$ therapy to death. Second-line OS (OS2) was defined as the survival time from the start of 2L therapy to death.

Baseline characteristics and efficacy data for patients who received any $2 \mathrm{~L}$ or no $2 \mathrm{~L}$ therapy were derived from the treated population in the $1 \mathrm{~L}$ setting. Three multivariate analyses were carried out to evaluate factors associated with (1) total OS, (2) post-1L OS (to understand the effect of $2 \mathrm{~L}$ treatment), and (3) OS2 (only in patients who received $2 \mathrm{~L}$ treatment). Each employed a Cox proportional hazards model to evaluate association of factors with OS. The multivariate analysis for total OS evaluated effects of $1 \mathrm{~L}$ treatment, $2 \mathrm{~L}$ treatment, and the following prognostic factors at baseline: geographic region (North America vs other), age, Karnofsky performance status (KPS, 70-80 vs 90-100), neutrophil-to-lymphocyte ratio (NLR, $\leqslant 5 \quad v s>5)$, pancreatic cancer primary location (head $v s$ body/tail), stage at initial diagnosis, carbohydrate antigen 19-9 (CA19-9; evaluated as a continuous variable), presence of liver metastases, presence of peritoneal carcinomatosis, previous Whipple procedure, presence of biliary stent, presence of pulmonary metastases, and number of metastatic sites. The analysis for post-1L OS evaluated the effects of $1 \mathrm{~L}$ treatment (nab-P + Gem vs Gem), 2L treatment (yes vs no), 1L progression-free survival (PFS; longer $v s$ shorter than the median PFS of 4.4 months observed for the entire intention-to-treat (ITT) population), region (North America $v s$ other), number of metastatic sites at baseline, and the following factors at the end of $1 \mathrm{~L}$ treatment: age, KPS (70-80 vs $\leqslant 60$ and $90-100 v s \leqslant 60)$, and NLR $(\leqslant 5 v s>5)$. The analysis of OS2 evaluated effects of $1 \mathrm{~L}$ treatment (nab-P + Gem vs Gem), 1L PFS (longer vs shorter than the median of 4.4 months observed for the entire ITT population), type of $2 \mathrm{~L}$ treatment (combination $v s$ monotherapy), region (North America $v s$ other), number of metastatic sites at baseline, and the following factors at the end of 1L treatment: age, KPS (70-80 vs $\leqslant 60$ and $90-100 v s \leqslant 60)$, and NLR $(\leqslant 5 v s>5)$. For each analysis, a stepwise procedure was performed, to evaluate the treatment effect and identify the possible factors associated with OS. A significance level of 0.20 was required for entry into the model. A significance level of 0.10 was required to remain in the model.

Both MPACT and the observational extension of the MPACT study (NCT02021500) were conducted in accordance with the amended Declaration of Helsinki. The local institutional review boards or independent ethics committees approved the protocol and written informed consent was obtained from all patients.

\section{RESULTS}

Patients and $2 \mathrm{~L}$ treatments. Patient enrolment in MPACT occurred between May 2009 and April 2012 (ITT population: $n=431$ for $n a b-\mathrm{P}+\mathrm{Gem}$ and $n=430$ for Gem alone). A total of 823 of these 861 patients were treated: 421 with $n a b-\mathrm{P}+\mathrm{Gem}$ and 402 with Gem alone. After the MPACT trial closed, 45 surviving patients continued to be followed in the observational extension study ( $n=26$ for $n a b-\mathrm{P}+\mathrm{Gem}$ and $n=19$ for Gem alone). The numbers of patients who received $2 \mathrm{~L}$ treatment were similar between treatment arms ( $n=170$ of $421(40 \%)$ for $n a b-P+G e m$ and 177 of 402 (44\%) for Gem alone). Most of the $2 \mathrm{~L}$ regimens contained a fluoropyrimidine (5-FU or capecitabine): 132 of 170 $(78 \%)$ in the $n a b-\mathrm{P}+\mathrm{Gem}$ arm and 135 of 177 (76\%) in the Gemalone arm; the majority of these were combination treatments (98 of $132(74 \%)$ and 107 of 135 (79\%), respectively), with the remainder being fluoropyrimidine monotherapy. In addition, 11 patients who received 1L Gem alone received nab-P monotherapy as $2 \mathrm{~L}$ treatment $(26 \%$ of the 'other than fluoropyrimidinecontaining' category) and 7 patients received nab-P + Gem. Of the 347 patients who received a $2 \mathrm{~L}$ therapy, 69 (20\%) received a third-line therapy (29 in the $n a b-\mathrm{P}+\mathrm{Gem}$ arm and 40 in the Gem-alone arm).

Baseline characteristics of patients who received $2 \mathrm{~L}$ treatment were generally well balanced at the time of initial randomisation to $1 \mathrm{~L}$ treatment (Table 1). However, compared with the ITT population, a higher percentage of patients who received $2 \mathrm{~L}$ therapy had a KPS of $90-100$ (68\% for patients who received 1L $n a b-\mathrm{P}+\mathrm{Gem}$ and a $2 \mathrm{~L}$ therapy, and $75 \%$ for patients who received $1 \mathrm{~L}$ Gem alone and a $2 \mathrm{~L}$ therapy $v s 58 \%$ and $62 \%$, respectively, in the ITT population). Baseline characteristics were also evaluated by specific $2 \mathrm{~L}$ treatment received (Supplementary Table 1). Patient characteristics were relatively well balanced among the subgroups, 
except that patients in the $n a b-\mathrm{P}+\mathrm{Gem}$ and Gem-alone arms, who received 2L FOLFIRINOX ( $n=18$ and 17 , respectively), when compared with those in the ITT arms, were younger (median ages, 53.5 and 56.0 vs 62.0 and 63.0 years), fitter (KPS 90-100, 72\% and $76 \%$ vs $58 \%$ and $62 \%$ ), and less likely to have a higher tumour burden ( $>3$ metastatic sites; 0 and $12 \%$ vs $14 \%$ and $15 \%$ ); however, baseline CA19-9 was similar or higher in patients who received $2 \mathrm{~L}$ FOLFIRINOX $v s$ the ITT population (median 5539 and $2368 \mathrm{U} \mathrm{ml}^{-1}$ vs 2294 and $2759 \mathrm{U} \mathrm{ml}^{-1}$ ).
As MPACT was designed as a prospective 1L study, no information was collected on patient characteristics at the start of $2 \mathrm{~L}$ therapy. However, as a surrogate, a few key patient characteristics at the end of $1 \mathrm{~L}$ treatment, such as KPS, CA19-9, and NLR, are reported in Table 2, to help understand some patient characteristics of those who received a subsequent therapy.

Among patients who received $2 \mathrm{~L}$ therapy, most discontinued $1 \mathrm{~L}$ treatment due to disease progression $(59 \%$ and $74 \%$ in the $n a b-\mathrm{P}+\mathrm{Gem}$ and Gem-alone arms, respectively). Fewer patients

Table 1. Baseline characteristics at time of $1 \mathrm{~L}$ randomisation

\begin{tabular}{|c|c|c|c|c|c|c|}
\hline & \multicolumn{2}{|c|}{ ITT } & \multicolumn{2}{|c|}{ Any $2 \mathrm{~L}$ treatment } & \multicolumn{2}{|c|}{ No 2L treatment } \\
\hline & nab-P + Gem & Gem & nab-P + Gem & Gem & nab-P + Gem & Gem \\
\hline$n$ & 431 & 430 & $170^{a}$ & $177^{a}$ & $250^{a}$ & $226^{a}$ \\
\hline Age, median, years & 62.0 & 63.0 & 61.0 & 62.0 & 63.0 & 64.0 \\
\hline $\begin{array}{l}\text { KPS, \% } \\
\text { 90-100 } \\
70-80\end{array}$ & $\begin{array}{l}58 \\
42\end{array}$ & $\begin{array}{l}62 \\
38\end{array}$ & $\begin{array}{l}68 \\
32\end{array}$ & $\begin{array}{l}75 \\
25\end{array}$ & $\begin{array}{l}52 \\
48\end{array}$ & $\begin{array}{l}51^{b} \\
49^{b}\end{array}$ \\
\hline $\begin{array}{l}\text { CA19-9 } \\
\qquad \begin{array}{l}\text { U ml } \\
\text {-1, median } \\
\geqslant 59 \times \text { ULN, \% }\end{array}\end{array}$ & $\begin{array}{c}n=379 \\
2294 \\
52\end{array}$ & $\begin{array}{c}n=371 \\
2759 \\
53\end{array}$ & $\begin{array}{c}n=152 \\
2644 \\
49\end{array}$ & $\begin{array}{c}n=162 \\
2096 \\
46\end{array}$ & $\begin{array}{c}n=225 \\
1951 \\
45\end{array}$ & $\begin{array}{c}n=206 \\
3733 \\
50\end{array}$ \\
\hline $\begin{array}{l}\text { Region, \% } \\
\text { North America } \\
\text { Other }\end{array}$ & $\begin{array}{l}62 \\
38\end{array}$ & $\begin{array}{l}63 \\
38\end{array}$ & $\begin{array}{l}66 \\
34\end{array}$ & $\begin{array}{l}63 \\
37\end{array}$ & $\begin{array}{l}59 \\
41\end{array}$ & $\begin{array}{l}60 \\
40\end{array}$ \\
\hline $\begin{array}{l}\text { No. metastatic sites, } \% \\
\begin{array}{l}1-3 \\
>3\end{array}\end{array}$ & $\begin{array}{l}86 \\
14\end{array}$ & $\begin{array}{l}85 \\
15\end{array}$ & $\begin{array}{l}88 \\
12\end{array}$ & $\begin{array}{l}88 \\
12\end{array}$ & $\begin{array}{l}85 \\
15\end{array}$ & $\begin{array}{l}84 \\
16\end{array}$ \\
\hline $\begin{array}{l}\text { NLR, \% } \\
\quad \leqslant 5 \\
>5\end{array}$ & $\begin{array}{c}n=426 \\
62 \\
38\end{array}$ & $\begin{array}{c}n=426 \\
65 \\
35\end{array}$ & $\begin{array}{l}75 \\
25\end{array}$ & $\begin{array}{l}73 \\
27\end{array}$ & $\begin{array}{l}55 \\
45\end{array}$ & $\begin{array}{l}58 \\
42\end{array}$ \\
\hline \multicolumn{7}{|c|}{$\begin{array}{l}\text { Abbreviations: } 1 \mathrm{~L}=\text { first-line; } 2 \mathrm{~L}=\text { second-line; } \mathrm{CA} 19-9=\text { carbohydrate antigen 19-9; Gem = gemcitabine; ITT=intention-to-treat; KPS = Karnofsky performance status; nab-P = nab-paclitaxel; } \\
\mathrm{NLR}=\text { neutrophil-to-lymphocyte ratio; } U L N=\text { upper limit of normal. }\end{array}$} \\
\hline
\end{tabular}

Table 2. Patient characteristics at end of $1 \mathrm{~L}$ treatment by $2 \mathrm{~L}$ therapy received

\begin{tabular}{|c|c|c|c|c|c|c|c|c|c|c|c|c|c|c|c|c|}
\hline & \multicolumn{2}{|c|}{$\begin{array}{c}\text { Any 2L } \\
\text { treatment }\end{array}$} & \multicolumn{2}{|c|}{$\begin{array}{c}\text { No } 2 \mathrm{~L} \\
\text { treatment }\end{array}$} & \multicolumn{2}{|c|}{$\begin{array}{l}\text { Fluoro- } \\
\text { pyrimidine- } \\
\text { containing }\end{array}$} & \multicolumn{2}{|c|}{$\begin{array}{l}\text { Fluoro- } \\
\text { pyrimidine } \\
\text { combo }\end{array}$} & \multicolumn{2}{|c|}{ FOLFIRINOX } & \multicolumn{2}{|c|}{ FOLFOX/OFF } & \multicolumn{2}{|c|}{$\begin{array}{l}\text { Fluoro- } \\
\text { pyrimidine } \\
\text { mono }\end{array}$} & \multicolumn{2}{|c|}{$\begin{array}{l}\text { Other (than } \\
\text { fluoro- } \\
\text { pyrimidine- } \\
\text { containing) }^{\text {a }}\end{array}$} \\
\hline & $\begin{array}{c}\text { nab-P + } \\
\text { Gem }\end{array}$ & Gem & $\begin{array}{c}\text { nab-P }+ \\
\text { Gem }\end{array}$ & Gem & $\begin{array}{c}\text { nab-P }+ \\
\text { Gem }\end{array}$ & Gem & $\begin{array}{c}\text { nab-P }+ \\
\text { Gem }\end{array}$ & Gem & $\begin{array}{c}\text { nab-P }+ \\
\text { Gem }\end{array}$ & Gem & $\begin{array}{c}\text { nab-P }+ \\
\text { Gem }\end{array}$ & Gem & $\begin{array}{c}\text { nab-P }+ \\
\text { Gem }\end{array}$ & Gem & $\begin{array}{c}\text { nab-P }+ \\
\text { Gem }\end{array}$ & Gem \\
\hline$n$ & 170 & 177 & 250 & 226 & 132 & 135 & 98 & 107 & 18 & 17 & 36 & 49 & 34 & 28 & 38 & 42 \\
\hline Age, median, years & 61.8 & 62.5 & 63.6 & 65.2 & 60.4 & 62.5 & 59.8 & 62.5 & 54.4 & 56.2 & 59.5 & 64.7 & 62.5 & 61.3 & 65.9 & 62.2 \\
\hline $\begin{array}{l}\text { KPS } \\
n \\
90-100, \% \\
80, \% \\
70, \% \\
\leqslant 60, \%\end{array}$ & $\begin{array}{l}170 \\
43 \\
37 \\
11 \\
9\end{array}$ & $\begin{array}{c}176 \\
48 \\
34 \\
10 \\
8\end{array}$ & $\begin{array}{l}238 \\
26 \\
28 \\
19 \\
27\end{array}$ & $\begin{array}{c}219 \\
22 \\
31 \\
19 \\
28\end{array}$ & $\begin{array}{c}132 \\
43 \\
38 \\
8 \\
11\end{array}$ & $\begin{array}{c}135 \\
45 \\
35 \\
12 \\
8\end{array}$ & $\begin{array}{c}98 \\
47 \\
38 \\
6 \\
9\end{array}$ & $\begin{array}{c}107 \\
48 \\
35 \\
11 \\
7\end{array}$ & $\begin{array}{c}18 \\
50 \\
39 \\
6 \\
6\end{array}$ & $\begin{array}{c}17 \\
59 \\
12 \\
29 \\
0\end{array}$ & $\begin{array}{c}36 \\
44 \\
31 \\
6 \\
19\end{array}$ & $\begin{array}{c}49 \\
35 \\
51 \\
10 \\
4\end{array}$ & $\begin{array}{l}34 \\
32 \\
38 \\
15 \\
15\end{array}$ & $\begin{array}{l}28 \\
36 \\
36 \\
14 \\
14\end{array}$ & $\begin{array}{c}38 \\
42 \\
34 \\
21 \\
3\end{array}$ & $\begin{array}{c}41 \\
56 \\
32 \\
5 \\
7\end{array}$ \\
\hline $\begin{array}{l}\text { CA19-9 } \\
n \\
\cup \mathrm{ml}^{-1}, \text { median } \\
\geqslant 59 \times \text { ULN, \% }\end{array}$ & $\begin{array}{c}144 \\
276 \\
26\end{array}$ & $\begin{array}{c}131 \\
380 \\
32\end{array}$ & $\begin{array}{c}168 \\
246 \\
29\end{array}$ & $\begin{array}{c}120 \\
1674 \\
45\end{array}$ & $\begin{array}{c}112 \\
276 \\
28\end{array}$ & $\begin{array}{c}99 \\
514 \\
33\end{array}$ & $\begin{array}{c}84 \\
241 \\
27\end{array}$ & $\begin{array}{c}76 \\
547 \\
33\end{array}$ & $\begin{array}{c}16 \\
195 \\
19\end{array}$ & $\begin{array}{c}10 \\
401 \\
20\end{array}$ & $\begin{array}{c}32 \\
398 \\
28\end{array}$ & $\begin{array}{c}33 \\
261 \\
30\end{array}$ & $\begin{array}{c}28 \\
289 \\
29\end{array}$ & $\begin{array}{c}23 \\
148 \\
35\end{array}$ & $\begin{array}{c}32 \\
277 \\
19\end{array}$ & $\begin{array}{c}32 \\
302 \\
28\end{array}$ \\
\hline NLR $\leqslant 5, \%$ & 74 & 67 & 59 & 44 & 77 & 64 & 77 & 65 & 72 & 71 & 69 & 63 & 79 & 61 & 61 & 76 \\
\hline $\begin{array}{l}\text { Abbreviations: } 1 \mathrm{~L}=\text { first } \\
\text { and oxaliplatin; Gem = } \\
U L N=\text { upper limit of } n \\
a_{\text {Regimens in the Othe }} \\
\text { Gem-based combinatic }\end{array}$ & $\begin{array}{l}\text { e; } 2 \mathrm{~L}=\mathrm{se} \\
\text { mcitabine } \\
\text { al. } \\
\text { ategory in }\end{array}$ & $\begin{array}{l}\text { ond-lin } \\
\text { KPS }= \\
\text { cluded }\end{array}$ & $\begin{array}{l}\text { A19-9= } \\
\text { nofsky } P \\
\text { following }\end{array}$ & $\begin{array}{l}\text { ohy } \\
\text { ab-P }\end{array}$ & $\begin{array}{l}\text { rate antige } \\
\text { ce status; } \\
\text { Gem (for }\end{array}$ & $\begin{array}{l}\text { 9; FOL } \\
\text { P nak } \\
\text { L Gen }\end{array}$ & $\begin{array}{l}\text { IRINOX }=f \\
\text { paclitaxel; } \\
\text { arm only), }\end{array}$ & nab- & $\begin{array}{l}\text { d, 5-fluorour } \\
\text { utrophil-to- } \\
\text { nnotherapy }\end{array}$ & $\begin{array}{l}\text { cil, irin } \\
\text { mpho } \\
\text { or the }\end{array}$ & $\begin{array}{l}\text { tecan, and } \\
\text { yte ratio; O } \\
1 \mathrm{~L} \text { Gem arm }\end{array}$ & $\begin{array}{l}\text { xalip } \\
F=0 \\
\text { only) }\end{array}$ & $\begin{array}{l}\text {; FOLFC } \\
\text { platin, } f \\
\text { otinib-c }\end{array}$ & folir & $\begin{array}{l}\text { cid, 5-fluo } \\
\text { and 5-fluo } \\
\text { gimens, ar }\end{array}$ & $\begin{array}{l}\text { uracil, } \\
\text { uracil; } \\
\text { other }\end{array}$ \\
\hline
\end{tabular}


(26\% and $14 \%$ of patients in the $n a b-\mathrm{P}+\mathrm{Gem}$ and Gem-alone arms, respectively) discontinued $1 \mathrm{~L}$ treatment due to adverse events. The median durations of $1 \mathrm{~L}$ therapy in patients who received $2 \mathrm{~L}$ therapy were 5.3 months for $n a b-\mathrm{P}+\mathrm{Gem}$ and 3.7 months for Gem alone (Supplementary Figure S1).

Efficacy in the 1L setting. Pooling both $1 \mathrm{~L}$ treatment arms, the median total OS from 1L randomisation for patients who did $(n=347)$ and did not $(n=476)$ receive a $2 \mathrm{~L}$ therapy was 10.9 and 5.4 months, respectively. In the cohort of patients who received any $2 \mathrm{~L}$ therapy, efficacy was greater with $n a b-\mathrm{P}+\mathrm{Gem} v s \mathrm{Gem}$ alone in terms of total OS from $1 \mathrm{~L}$ randomisation (median $=12.8$ vs 9.9 months, $\mathrm{HR}=0.76,95 \% \mathrm{CI}=0.61-0.95, \quad P=0.015$; Figures 1 and 2) and overall response rate during $1 \mathrm{~L}$ therapy $(28 \%$ vs $9 \%, P<0.001)$. First-line PFS was longer for patients who received $1 \mathrm{~L} n a b-\mathrm{P}+\mathrm{Gem} v s$ Gem alone, although the difference was not statistically significant (median $=7.6$ vs 5.4 months, $\mathrm{HR}=0.75, \quad 95 \% \quad \mathrm{CI}=0.55-1.02, \quad P=0.067 ; \quad$ Supplementary Table 2). For patients who did not receive $2 \mathrm{~L}$ therapy, median OS was 6.3 vs 4.3 months $(\mathrm{HR}=0.64,95 \% \mathrm{CI}=0.53-0.78$, $P<0.001)$ and overall response rate during $1 \mathrm{~L}$ treatment was $21 \%$ vs $7 \%(P<0.001)$, respectively.

In the cohort of patients who received $2 \mathrm{~L}$ therapy, a lower percentage of patients in the nab-P + Gem vs Gem-alone arm experienced progressive disease before week 8 (9\% vs 15\%, respectively). In the cohort of patients who did not receive $2 \mathrm{~L}$ therapy, $6 \%$ of those who received $1 \mathrm{~L} n a b-\mathrm{P}+\mathrm{Gem} v \mathrm{~s} 12 \%$ of those who received Gem alone had progressive disease before week 8. To further eliminate potential bias of poor prognostic factors in the group of patients who did not receive $2 \mathrm{~L}$ therapy, total OS was examined in a pooled-treatment-arm cohort of only those patients with KPS $90-100$ and NLR $\leqslant 5$ at the end of $1 \mathrm{~L}$ treatment.

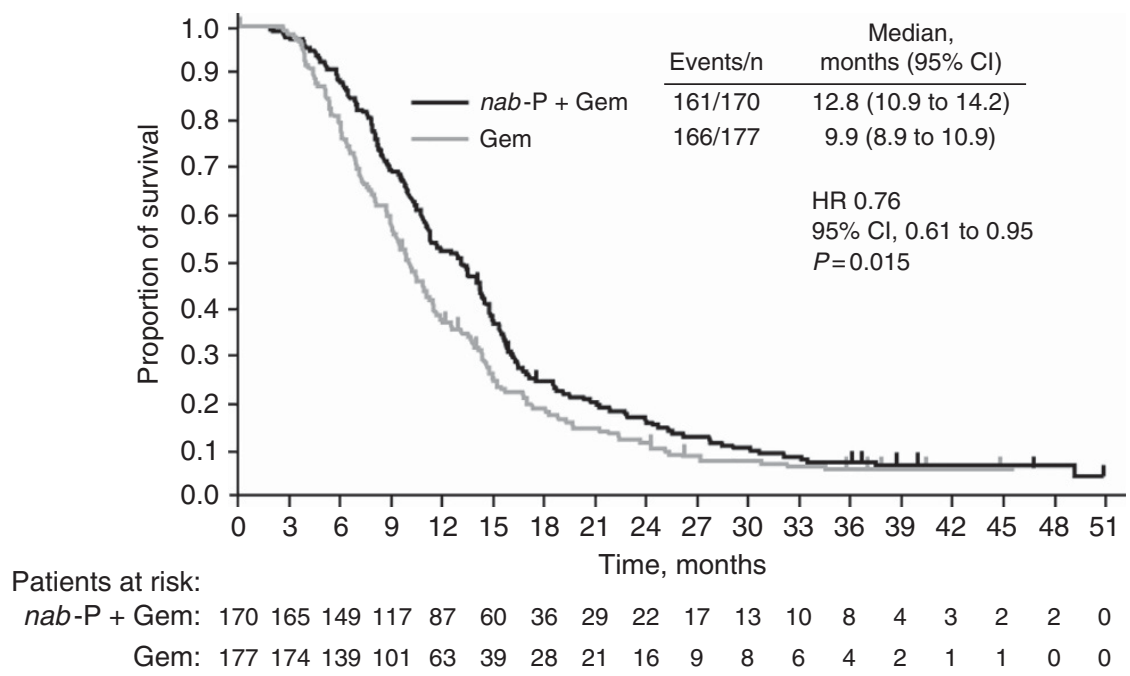

Figure 1. Total OS in patients who received $2 \mathrm{~L}$ therapy. Gem = gemcitabine; $\mathrm{HR}=$ hazard ratio; nab-P=nab-paclitaxel.

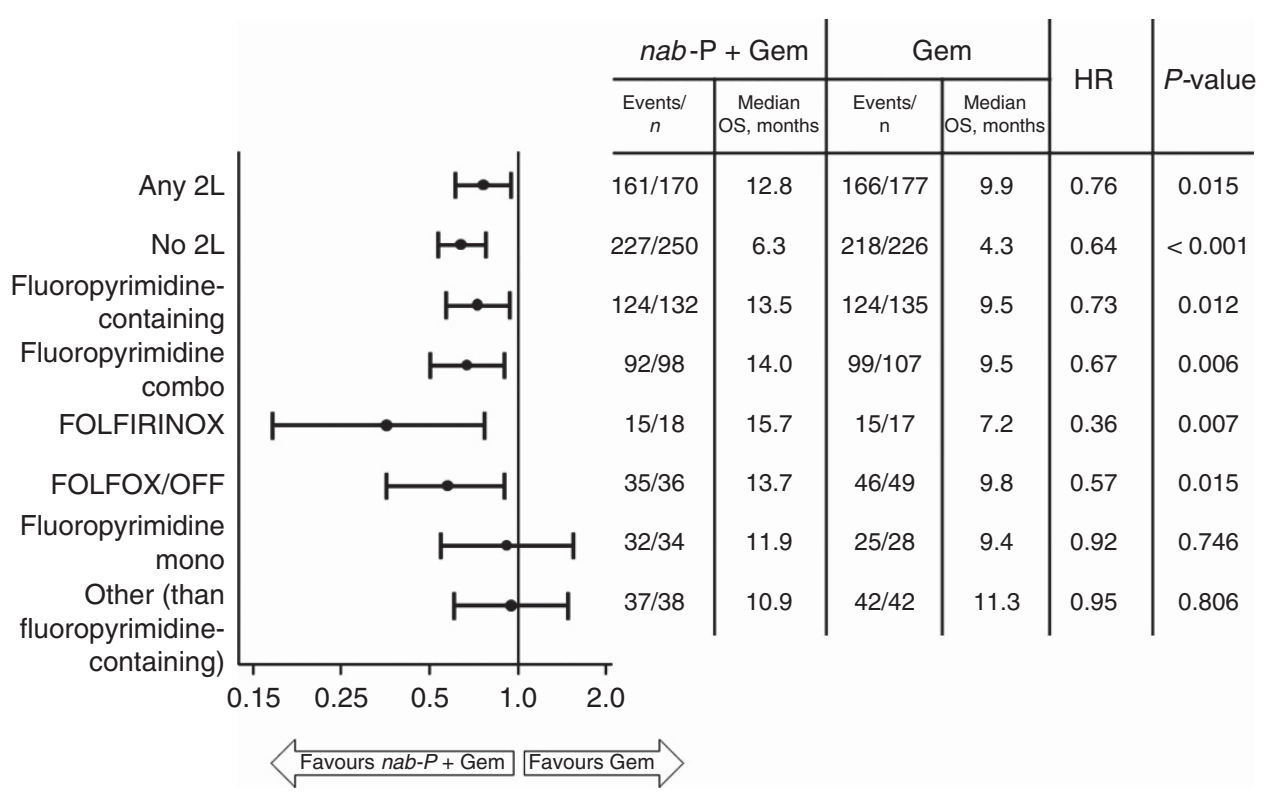

Figure 2. Forest plot of total OS in subgroups defined by $2 \mathrm{~L}$ therapy. FOLFIRINOX = folinic acid, 5-fluorouracil, irinotecan, and oxaliplatin; FOLFOX = folinic acid, 5-FU, and oxaliplatin; Gem = gemcitabine; HR= hazard ratio; mono = monotherapy; nab-P = nab-paclitaxel;

OFF = oxaliplatin, folinic acid, and 5-FU. 
Table 3. Multivariate analyses of total OS, post-1L OS, and survival time from the start of $2 \mathrm{~L}$ therapy to death (OS2)

\begin{tabular}{|c|c|c|}
\hline Covariate $^{a}$ & HR $(95 \% \mathrm{Cl})$ & $P$-va \\
\hline \multicolumn{3}{|c|}{$\begin{array}{l}\text { Total OS (includes treated patients with or without a } 2 L \text { therapy } \\
n=741 \text { ) }\end{array}$} \\
\hline 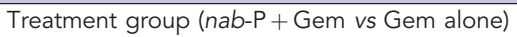 & $0.63(0.54-0.74)$ & \\
\hline & & \\
\hline & & \\
\hline $\mathrm{KP}$ & & \\
\hline & 1.5 & \\
\hline & & \\
\hline e at baseline $(<65 \mathrm{vs} \geqslant 65$ years & $0.88(0.75-1.02)$ & \\
\hline
\end{tabular}

Post-1L OS (includes treated patients with or without a $2 \mathrm{~L}$ therapy; $\boldsymbol{n}=793$ )

\begin{tabular}{|c|c|c|}
\hline $\begin{array}{l}\text { Treatment group (nab-P }+ \text { Gem vs Gem alone) } \\
2 L \text { therapy (with vs without) } \\
\text { NLR at end of } 1 L(\leqslant 5 \text { vs }>5)\end{array}$ & $\begin{array}{l}0.73(0.63-0.85) \\
0.47(0.40-0.54) \\
0.60(0.52-0.70)\end{array}$ & $\begin{array}{l}<0.001 \\
<0.001 \\
<0.001\end{array}$ \\
\hline $\begin{array}{l}\text { KPS at end of } 1 \mathrm{~L} \\
90-100 \text { vs } \leqslant 60 \\
70-80 \text { vs } \leqslant 60\end{array}$ & $\begin{array}{l}0.46(0.37-0.57) \\
0.57(0.47-0.70)\end{array}$ & $\begin{array}{l}<0.001 \\
<0.001\end{array}$ \\
\hline $\begin{array}{l}\text { PFS, months ( } \geqslant 4.4 \text { vs }<4.4)^{\mathrm{b}} \\
\text { Geographic region (North America vs others) }\end{array}$ & $\begin{array}{l}0.78(0.67-0.91) \\
0.86(0.74-1.00)\end{array}$ & $\begin{array}{l}0.002 \\
0.051\end{array}$ \\
\hline \multicolumn{3}{|c|}{ OS2 (only patients who received $2 \mathrm{~L}$ therapy; $n=346$ ) } \\
\hline $\begin{array}{l}\text { Number of metastatic sites } \\
\text { NLR at end of } 1 \mathrm{~L}(\leqslant 5 \text { vs }>5)\end{array}$ & $\begin{array}{l}1.15(1.02-1.29) \\
0.76(0.60-0.97)\end{array}$ & $\begin{array}{l}0.018 \\
0.027\end{array}$ \\
\hline $\begin{array}{l}\text { KPS at end of } 1 \mathrm{~L} \\
90-100 \text { vs } \leqslant 60 \\
70-80 \text { vs } \leqslant 60\end{array}$ & $\begin{array}{l}0.53(0.35-0.81) \\
0.66(0.44-1.00)\end{array}$ & $\begin{array}{l}0.003 \\
0.052\end{array}$ \\
\hline
\end{tabular}

Abbreviations: $1 \mathrm{~L}=$ first-line; $2 \mathrm{~L}=$ second-line; $\mathrm{CA1} 19-9=$ carbohydrate antigen $19-9 ; \mathrm{Cl}=$ confidence interval; Gem = gemcitabine; $\mathrm{HR}=$ hazard ratio; $\mathrm{KPS}=$ Karnofsky performance status; nab-P = nab-paclitaxel; NLR = neutrophil-to-lymphocyte ratio; $O S=$ overall survival; $\mathrm{PFS}=$ progression-free survival.

${ }^{a}$ Covariates tested for each multivariate analysis are listed in the Patients and Methods section.

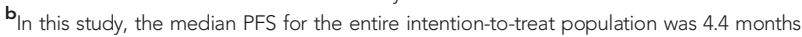

Overall survival was longer for those who did $(n=116) v s$ did not $(n=70)$ receive $2 \mathrm{~L}$ therapy: median OS 14.1 vs 9.6 months $(\mathrm{HR}=0.72,95 \% \mathrm{CI}=0.52-0.99, P=0.042)$.

Baseline factors found to significantly associate with longer total OS in a multivariate analysis included $1 \mathrm{~L}$ treatment with nab-P + Gem, use of any 2L therapy, better KPS at baseline (90-100 vs $70-80)$, no liver metastases at baseline, NLR at baseline $\leqslant 5 v s>5$ $(P<0.001$ for each), and lower CA19-9 at baseline (evaluated as a continuous variable, $P=0.005$; Table 3 ).

The total OS in the group of patients who received a fluoropyrimidine-containing regimen was significantly longer for those who received $1 \mathrm{~L} n a b-\mathrm{P}+\mathrm{Gem} v s$ Gem alone (median $=13.5$ vs 9.5 months, $\mathrm{HR}=0.73, \quad 95 \% \quad \mathrm{CI}=0.57-0.93, \quad P=0.012$; Figure 2). The longest median total OS values in the nab-P $+\mathrm{Gem}$ arm were for patients who received fluoropyrimidine-containing combinations, such as FOLFIRINOX $(n=18, \quad$ median $=15.7$ months) and FOLFOX (folinic acid, 5-FU, and oxaliplatin; $n=36$, median $=13.7$ months); 2 patients who received $2 \mathrm{~L}$ FOLFIRI (folinic acid, 5-FU, and irinotecan) after nab-P + Gem had a total OS of 5.8 and 8.9 months.

A minority of patients $(23 \%)$ received $2 \mathrm{~L}$ therapies other than fluoropyrimidine-containing regimens; these included erlotinib and various investigational agents. In addition, some patients in the $1 \mathrm{~L}$ Gem-alone arm received a nab-P-based regimen for $2 \mathrm{~L}$ treatment. Median total OS for patients who received $1 \mathrm{~L} \mathrm{Gem}$ alone and 2L nab-P monotherapy $(n=11)$ or $n a b-\mathrm{P}+\operatorname{Gem}(n=7)$ was 8.8 and 7.9 months, respectively.

Post-1L survival (from end of 1L therapy to death). To understand the differences between outcomes in patients who did $v s$ did not receive a $2 \mathrm{~L}$ therapy, the duration of survival from the end of $1 \mathrm{~L}$ therapy was examined. A multivariate analysis

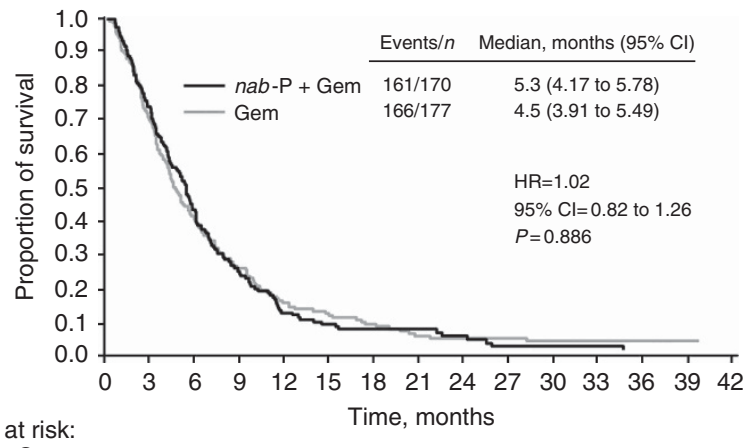

Patients at risk:

nab-P + Gem: $170 \begin{array}{llllllllllllll}122 & 68 & 36 & 16 & 11 & 7 & 6 & 4 & 1 & 1 & 1 & 0 & 0 & 0\end{array}$ Gem: $177 \begin{array}{llllllllllllll}122 & 67 & 41 & 23 & 16 & 12 & 6 & 4 & 4 & 3 & 2 & 1 & 1 & 0\end{array}$

Figure 3. Second-line OS (OS2), defined as survival time from the start of $2 \mathrm{~L}$ therapy to death, in patients who received $2 \mathrm{~L}$ therapy.

$\mathrm{Gem}=$ gemcitabine; $\mathrm{HR}=$ hazard ratio; nab-P = nab-paclitaxel.

including treated patients in MPACT identified the following factors significantly associated with longer post-1L survival: 1L treatment with nab-P + Gem, use of any $2 \mathrm{~L}$ therapy, NLR $\leqslant 5$ $v s>5$ at end of $1 \mathrm{~L}$ therapy, better KPS at end of 1L therapy (90$100 v s \leqslant 60$ and $70-80 v s \leqslant 60 ; P<0.001$ for each), and $1 \mathrm{~L}$ PFS $\geqslant 4.4$ months $v s<4.4$ months (the median for the ITT population, $P=0.002$; Table 3).

OS2: survival time from the start of $2 \mathrm{~L}$ therapy to death. For patients who received any 2L therapy $(n=347)$, OS2 was similar for patients who received $1 \mathrm{~L}$ nab-P $+\mathrm{Gem}$ vs Gem alone (median $=5.3$ months for nab-P + Gem vs 4.5 months for Gem alone; all $2 \mathrm{~L}$ treatments; $\mathrm{HR}$ for $\mathrm{OS}=1.02,95 \% \mathrm{CI}=0.82-1.26$, $P=0.886$; Figure 3 and Supplementary Table 3 ). Among patients who received a fluoropyrimidine-containing $2 \mathrm{~L}$ treatment, median OS2 was 5.7 months for $n a b-\mathrm{P}+\mathrm{Gem} v s 4.5$ months for Gem alone $(\mathrm{HR}$ for $\mathrm{OS}=0.94, \quad 95 \% \quad \mathrm{CI}=0.73-1.20, \quad P=0.606$; Supplementary Table 3).

Among patients who received any 2L therapy, factors found to significantly associate with longer OS2 in a multivariate analysis included fewer metastatic sites at baseline, NLR $\leqslant 5$ at end of $1 \mathrm{~L}$ treatment, and KPS $90-100 v s \leqslant 60$ at end of $1 \mathrm{~L}$ treatment $(P<0.05$ for each; Table 3$)$.

In general, groups with longer median $1 \mathrm{~L}$ treatment duration demonstrated a trend towards longer median OS2 (Supplementary Figure 1).

\section{DISCUSSION}

The overall results of this analysis demonstrated that $2 \mathrm{~L}$ treatment was feasible and appeared to confer an OS benefit compared with no $2 \mathrm{~L}$ treatment in the large, randomised MPACT patient population. Indeed, the median OS of patients in the nab-P + Gem group who received a $2 \mathrm{~L}$ treatment was over 4 months longer than the median OS with nab-P + Gem in the ITT population (12.8 vs 8.7 months; Von Hoff et al, 2013) and over 6 months longer than the median OS with nab-P + Gem in patients who did not receive $2 \mathrm{~L}$ therapy (12.8 vs 6.2 months). A similar effect was observed in the Gem-alone arm (median OS, 9.9 for those who received $1 \mathrm{~L}$ Gem alone and a $2 \mathrm{~L}$ treatment $v s 6.6$ months in the ITT population). A matched cohort analysis of patients with good prognostic factors (KPS $90-100$ and NLR $\leqslant 5$ ) at the end of $1 \mathrm{~L}$ treatment supported a benefit for $2 \mathrm{~L}$ treatment, as did multivariate analyses of total OS and post-1L survival, further confirming that subsequent therapy is beneficial. Patients who received nab-P + Gem vs Gem alone for 1L therapy followed by fluoropyrimidine combination therapy as the $2 \mathrm{~L}$ treatment achieved a median OS2 of 
6.0 vs 4.6 months $(\mathrm{HR}=0.87,95 \% \mathrm{CI}=0.66-1.16, P=0.352)$, consistent with the idea that more effective $1 \mathrm{~L}$ therapy may have an important role in achieving optimal benefit from specific subsequent therapies. In addition, these data suggest that fluoropyrimidine combination regimens are feasible as $2 \mathrm{~L}$ therapy after nab-P + Gem and confer better results than monotherapy after $1 \mathrm{~L}$ combination therapy.

The median durations of OS2 (all 2L therapies combined, 5.3 months for $n a b-\mathrm{P}+\mathrm{Gem}$ and 4.5 months for Gem alone) were comparable to those reported in the PRODIGE trial of FOLFIRINOX vs Gem as 1L therapy (4.4 months in both arms; Conroy et al, 2011). Combination 2L therapies in MPACT appeared to result in longer survival. Some factors that might explain this effect are the efficacy of the specific $2 \mathrm{~L}$ treatment and the underlying patient biology that allowed patients to receive that therapy. For example, FOLFIRINOX after nab-P + Gem resulted in the longest OS of all treatments analysed (this result must be interpreted with caution given the small sample size $(n=18)$ ); however, FOLFIRINOX was given to patients with favourable characteristics at the end of $1 \mathrm{~L}$ therapy (e.g., those with better performance status), making it difficult to determine how much of the longer survival was due to the efficacy of FOLFIRINOX $v$ s inherent patient factors. Indeed, separate multivariate analyses demonstrated significant associations of total OS and post-1L OS with $1 \mathrm{~L} n a b-\mathrm{P}+\mathrm{Gem} v s$ Gem alone, receipt of 2L therapy, and KPS and NLR (at baseline for total OS and at the end of 1L therapy for post-1L OS). A third multivariate analysis identified baseline number of metastatic sites, as well as NLR and KPS at the end of 1L treatment, as significantly associated with OS2. These clinical characteristics at the end of $1 \mathrm{~L}$ therapy will help clinicians identify which patients will achieve the greatest benefit from 2L therapy. More research to distinguish the extent of benefit of treatment as opposed to favourable prognostic biology is required.

Sequential treatment planning for metastatic pancreatic cancer is a new concept made possible by therapeutic advances such as $n a b-\mathrm{P}+\mathrm{Gem}$ and FOLFIRINOX. Disease management previously focused on palliation of symptoms after progression following initial therapy. However, the results from this study and others (Supplementary Table 4) suggest that care plans for patients with metastatic pancreatic cancer are evolving to include multiple lines of therapy (Conroy et al, 2011; Gill et al, 2014; Oettle et al, 2014; Portal et al, 2015; Wang-Gillam et al, 2015; Kobayashi et al, 2016; Schmidt et al, 2016). In addition, although little is known about interactions between specific $1 \mathrm{~L}$ and $2 \mathrm{~L}$ therapies in terms of overall efficacy, groups with longer median duration of $1 \mathrm{~L}$ therapy generally had longer median OS2 in this subanalysis (Supplementary Figure 1) and a multivariate analysis demonstrated that PFS duration in the $1 \mathrm{~L}$ setting was associated with post-1L survival. The association between $1 \mathrm{~L}$ PFS and post-1L survival is consistent with a previous study (Reni et al, 2008). Taken together, these findings highlight the benefit that a patient could theoretically derive from maximizing duration of $1 \mathrm{~L}$ therapy (in the absence of disease progression) through careful monitoring of patient tolerability and enacting dose modifications when necessary.

Genomic analyses may hold promise in guiding treatment selection based on tumour biology (Sausen et al, 2015) and, although there are often valid reasons for a patient not to be offered 2L therapy (e.g., toxicity and patient decisions), a broader assortment of options and information on potential benefit of specific treatment sequences will undoubtedly improve patient care.

Interpretation of these results is subject to a number of limitations. First, as a post-hoc analysis of a prospective 1L study, no measures were enacted to ensure controlled comparisons among groups with regard to $2 \mathrm{~L}$ therapy. Second, beyond the start date and description of components, details on $2 \mathrm{~L}$ regimens were not collected, resulting in a lack of information on doses, schedules, duration of $2 \mathrm{~L}$ therapy, disease progression, and tolerability of $2 \mathrm{~L}$ treatment. In addition, patient characteristics were not specifically recorded at the initiation of $2 \mathrm{~L}$ therapy, although certain key factors, including age, CA19-9, and NLR were collected at the end of $1 \mathrm{~L}$ therapy. Third, when considering the longer OS in patients who received $2 \mathrm{~L}$ treatment $v s$ the ITT population, it is important to note that this cohort, by definition, excluded patients who died too early to receive $2 \mathrm{~L}$ treatment. Fourth, there are no data from this analysis on patients who received $2 \mathrm{~L}$ liposomal irinotecan, which recently became the only approved 2L option for metastatic pancreatic cancer (Merrimack Pharmaceuticals, 2015), nor was irinotecan a highly used option (e.g., as a component of FOLFIRI) at the time of trial conduct. Most patients in the MPACT study received oxaliplatin- and fluoropyrimidine-containing combination treatments (e.g., FOLFOX or OFF (oxaliplatin, folinic acid, and 5-FU)), per established treatment recommendations at the time the study was conducted.

The findings from this analysis support the increasing use of $2 \mathrm{~L}$ therapy in patients with metastatic pancreatic cancer. Specifically, these results showed that a fluoropyrimidine-containing treatment after $1 \mathrm{~L} n a b-\mathrm{P}+\mathrm{Gem}$ or Gem alone may be an active treatment sequence with favourable clinical benefit. Further guidance for the use of $2 \mathrm{~L}$ therapy will depend on enhanced identification of biologic predictors of $2 \mathrm{~L}$ treatment benefit, development of more active regimens, and a better understanding of how an effective $1 \mathrm{~L}$ therapy may influence clinical benefit in subsequent treatments.

\section{ACKNOWLEDGEMENTS}

Sponsorship of this study and article processing charges were funded by Celgene Corporation, Summit, NJ, USA. Writing assistance was provided by John R. McGuire, $\mathrm{PhD}$, of MediTech Media, LLC, and was funded by Celgene Corporation. Biostatistical support was provided by Xiaobin Yuan, $\mathrm{PhD}$, of Celgene Corporation. Scientific support was provided by Susan Coughlin, $\mathrm{PhD}$, of Celgene Corporation. We are fully responsible for all content and editorial decisions for this manuscript.

\section{CONFLICT OF INTEREST}

EGC: research funding, consultant or advisory role, Celgene. DDVH: consultant or advisory role, honoraria, Celgene; research funding, HonorHealth. JT: consultant or advisory role, honoraria, Celgene. RE-M: consultant or advisory role, research funding, Celgene. WWM: research funding, Celgene. MR: consultant or advisory role, honoraria, research funding, Celgene. $\mathrm{MH}$ and RW: nothing to disclose. HL, JSL, VM, AR and BL: employment, stock ownership, Celgene. DG: research funding, Celgene, Pfizer; consultant or advisory role (unremunerated), Celgene, Pfizer.

\section{REFERENCES}

Abrams TA, Meyer G, Moloney J, Meyerhardt JA, Wolpin BW, Schrag D, Fuchs CS (2014) Patterns of chemotherapy (CT) use in a population-based US-wide cohort of patients (pts) with metastatic pancreatic cancer (MPC). J Clin Oncol 32(suppl 5): abstract 4131.

American Cancer Society (2015) Cancer Facts and Figures 2015. American Cancer Society: Atlanta, GA, USA.

Burris 3rd HA, Moore MJ, Andersen J, Green MR, Rothenberg ML, Modiano MR, Cripps MC, Portenoy RK, Storniolo AM, Tarassoff P, Nelson R, Dorr FA, Stephens CD, Von Hoff DD (1997) Improvements in survival and clinical benefit with gemcitabine as first-line therapy for patients with advanced pancreas cancer: a randomized trial. J Clin Oncol 15: 2403-2413. 
Conroy T, Desseigne F, Ychou M, Bouche O, Guimbaud R, Becouarn Y, Adenis A, Raoul JL, Gourgou-Bourgade S, de la Fouchardiere C, Bennouna J, Bachet JB, Khemissa-Akouz F, Pere-Verge D, Delbaldo C, Assenat E, Chauffert B, Michel P, Montoto-Grillot C, Ducreux M (2011) FOLFIRINOX versus gemcitabine for metastatic pancreatic cancer. $N$ Engl J Med 364: 1817-1825.

Gill S, Ko Y, Cripps C, Beaudoin A, Dhesy-Thind SK, Zulfiqar M, Salweski P, Do T, Cano PO, Lam W, Dowden SD, Grassin H, Stewart J, Moore MJ (2014) PANCREOX: a randomized phase 3 study of 5FU/LV with or without oxaliplatin for second-line advanced pancreatic cancer (APC) in patients (pts) who have received gemcitabine (GEM)-based chemotherapy (CT). J Clin Oncol 32(suppl 5): abstract 4022.

Kobayashi N, Tokuhisa M, Goto A, Endo I, Ichikawa Y (2016) Second-line chemotherapy by FOLFIRINOX with unresectable pancreatic cancer (phase I, II study). J Clin Oncol 34(suppl 4S): abstract 297.

Merrimack Pharmaceuticals (2015) Onivyde (irinotecan liposome injection) [package insert], Cambridge, MA, USA.

Oettle H, Riess H, Stieler JM, Heil G, Schwaner I, Seraphin J, Gorner M, Molle M, Greten TF, Lakner V, Bischoff S, Sinn M, Dorken B, Pelzer U (2014) Second-line oxaliplatin, folinic acid, and fluorouracil versus folinic acid and fluorouracil alone for gemcitabine-refractory pancreatic cancer: outcomes from the CONKO-003 trial. J Clin Oncol 32: 2423-2429.

Portal A, Pernot S, Tougeron D, Arbaud C, Bidault AT, de la Fouchardiere C, Hammel P, Lecomte T, Dreanic J, Coriat R, Bachet JB, Dubreuil O, Marthey L, Dahan L, Tchoundjeu B, Locher C, Lepere C, Bonnetain F, Taieb J (2015) Nab-paclitaxel plus gemcitabine for metastatic pancreatic adenocarcinoma after FOLFIRINOX failure: an AGEO prospective multicentre cohort. Br J Cancer 113: 989-995.

Rahib L, Smith BD, Aizenberg R, Rosenzweig AB, Fleshman JM, Matrisian LM (2014) Projecting cancer incidence and deaths to 2030: the unexpected burden of thyroid, liver, and pancreas cancers in the United States. Cancer Res 74: 2913-2921.

Reni M, Berardi R, Mambrini A, Pasetto L, Cereda S, Ferrari VD, Cascinu S, Cantore M, Mazza E, Grisanti S (2008) A multi-centre retrospective review of second-line therapy in advanced pancreatic adenocarcinoma. Cancer Chemother Pharmacol 62: 673-678.

Sausen M, Phallen J, Adleff V, Jones S, Leary RJ, Barrett MT, Anagnostou V, Parpart-Li S, Murphy D, Kay Li Q, Hruban CA, Scharpf R, White JR, O’Dwyer PJ, Allen PJ, Eshleman JR, Thompson CB, Klimstra DS,
Linehan DC, Maitra A, Hruban RH, Diaz Jr LA, Von Hoff DD, Johansen JS, Drebin JA, Velculescu VE (2015) Clinical implications of genomic alterations in the tumour and circulation of pancreatic cancer patients. Nat Commun 6: 7686.

Schmidt SL, Durkal V, Jayavalsan SP, Thomas JP, Ritch PS, Erickson B, Christians KK, Tsai S, Evans DB, George B (2016) Outcomes in metastatic pancreatic adenocarcinoma (MPAC) patients treated with FOLFIRINOX (FFX)/FOLFOX(FX) and gemcitabine + nab-paclitaxel (NabG). J Clin Oncol 34(suppl 4S): abstract 397.

Smyth EN, Bapat B, Ball DE, Andre T, Kaye JA (2015) Metastatic pancreatic adenocarcinoma treatment patterns, health care resource use, and outcomes in France and the United Kingdom between 2009 and 2012: a retrospective study. Clin Ther 37: 1301-1316.

Surveillance, Epidemiology, and End Results Program (2016) SEER Stat Facts Sheets: Pancreas Cancer, Bethesda, MD, USA.

The National Comprehensive Cancer Network (2015) NCCN Clinical Practice Guidelines in Oncology: Pancreatic Adenocarcinoma, V2.2015, Washington, PA, USA.

Von Hoff DD, Ervin T, Arena FP, Chiorean EG, Infante J, Moore M, Seay T, Tjulandin SA, Ma WW, Saleh MN, Harris M, Reni M, Dowden S, Laheru D, Bahary N, Ramanathan RK, Tabernero J, Hidalgo M, Goldstein D, Van Cutsem E, Wei X, Iglesias J, Renschler MF (2013) Increased survival in pancreatic cancer with nab-paclitaxel plus gemcitabine. N Engl J Med 369: 1691-1703.

Wang-Gillam A, Li CP, Bodoky G, Dean A, Shan YS, Jameson G, Macarulla T, Lee KH, Cunningham D, Blanc JF, Hubner RA, Chiu CF, Schwartsmann G, Siveke JT, Braiteh F, Moyo V, Belanger B, Dhindsa N, Bayever E, Von Hoff DD, Chen LT. NAPOLI-1 Study Group (2015) Nanoliposomal irinotecan with fluorouracil and folinic acid in metastatic pancreatic cancer after previous gemcitabine-based therapy (NAPOLI-1): a global, randomised, open-label, phase 3 trial. Lancet 387(10018): 545-557.

World Health Organization (2015) GLOBOCAN 2012: Estimated Cancer Incidence, Mortality, and Prevalence Worldwide in 2012, Lyon, France (accessed 17 September 2015).

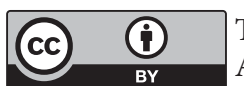

This work is licensed under the Creative Commons Attribution 4.0 International License. To view a copy of this license, visit http://creativecommons.org/licenses/by/4.0/

Supplementary Information accompanies this paper on British Journal of Cancer website (http://www.nature.com/bjc) 